\title{
Continuous-, derivative-, and differentiable-restrictions of measurable functions
}

by

\author{
Jack B. B row n (Auburn, Ala.)
}

\begin{abstract}
We review the known facts and establish some new results concerning continuous-restrictions, derivative-restrictions, and differentiable-restrictions of Lebesgue measurable, universally measurable, and Marczewski measurable functions, as well as functions which have the Baire properties in the wide and restricted senses. We also discuss some known examples and present a number of new examples to show that the theorems are sharp.
\end{abstract}

1. Introduction. "Measurability" means measurability with respect to one of the $\sigma$-algebras:

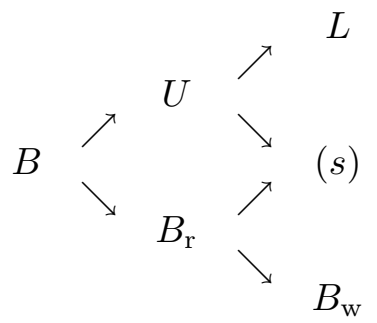

where $B=$ the Borel sets and $U, L$, and $(s)$ represent the universally measurable, Lebesgue measurable, and Marczewski measurable sets, respectively. $B_{\mathrm{r}}$ represents the sets with the Baire property (restricted sense) and $B_{\mathrm{w}}$ represents the sets with the Baire property (wide sense). The classes $B$, $L, B_{\mathrm{w}}$, and $B_{\mathrm{r}}$ are well known and definitions can be found in [10], and elsewhere. A set $M \in U$ if $M$ is measurable with respect to the completion of every Borel measure on the space [15]. A set $M \in(s)$ if every perfect subset of the space has a perfect subset which is a subset of or misses $M$ (see [15] and [19]). The properties $B, U,(s), B_{\mathrm{r}}$, and $B_{\mathrm{w}}$ have meaning in any complete separable metric space without isolated points, and $L$ has its usual meaning in the reals, $\mathbb{R}$. $\lambda$ will be used to denote Lebesgue measure.

The $\sigma$-ideals associated with these $\sigma$-algebras are the countable sets, the universal null sets, $U_{0}$, the Lebesgue null sets, $L_{0}$, the Marczewski null 
sets, $\left(s^{0}\right)$, the perfectly meagre or always first category sets, AFC, and the first category sets, FC. The $\sigma$-ideals consist of the sets, every subset of which belongs to the corresponding $\sigma$-algebra, and these classes are of course related as follows:

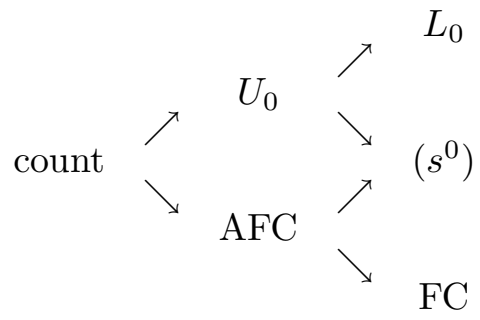

2. Lebesgue measurable functions. The best known theorem of the type we are interested in is the following:

Theorem 1. If $f: \mathbb{R} \rightarrow \mathbb{R}$ is L-measurable, then

1) there exists a non- $L_{0}$ set $M$ (indeed, for every $\varepsilon>0$, there exists $M$ with $\lambda\left(M^{c}\right)<\varepsilon$ ) such that $f \mid M$ is continuous [12],

2) there exists a co- $L_{0}$ set $M$ and a continuous (a.e. differentiable) $F$ : $\mathbb{R} \rightarrow \mathbb{R}$ such that $f\left|M=F^{\prime}\right| M[13]$,

3) there exists a perfect set $P$ such that $f \mid P$

(i) is monotonic [7],

(ii) is $C^{\infty}$ (relative to $P$ ) [11],

(iii) $=g \mid P$ for some $C^{1} g: \mathbb{R} \rightarrow \mathbb{R}[1]$.

Remarks. Parts 1) and 2) are sometimes referred to as "Lusin's 1st and 2nd Theorems". Part 3) was originally proved for continuous functions, but the proofs for the measurable case follow easily from part 1) and the results indicated in the references given.

EXAMPLE 1.1. It is well known that you cannot make the set $M$ of part 1) be co- $L_{0}$, even for a $B^{1}$ (Baire-1) function. For example, if $C_{1}, C_{2}, \ldots$ is a sequence of disjoint Cantor sets such that $\lambda\left[\left(C_{1} \cup C_{2} \cup \ldots\right)^{c}\right]=0$, and $f(x)=1 / n$ for $x \in C_{n}$, and $f(x)=0$ otherwise, then $f$ is such a $B^{1}$ function. This example also shows that you cannot make the set $M$ of part 1) be co- $\left(s^{0}\right)$ because if $M$ is co- $\left(s^{0}\right), M$ intersects every Cantor set. This means that $M$ would be dense in every $C_{n}$ and also dense in $\left(C_{1} \cup C_{2} \cup \ldots\right)^{c}$, so that $f \mid M$ could not be continuous at the elements of $M \cap C_{n}$.

EXAMPLE 1.2. It is known that you can make the set $M$ of part 1) be dense in $\mathbb{R}$ because of Blumberg's Theorem [2] but that you cannot make $M$ simultaneously dense and non- $L_{0}$ because of the example described above. Ceder has actually shown (see the proof of Example 2 of [6]) that you cannot 
make the set $M$ of part 1) simultaneously dense and of cardinality $c$ (you can obtain this for $B_{\mathrm{w}^{-}}$and ( $s$-measurable $f$, as we will see below).

EXAMPLE 1.3. It is known that you cannot make the set $M$ of part 1) non-FC, even (assuming $\mathrm{CH}$ ) for a $U$-measurable $f$ such as that described in Example 1 of [4]. That same example shows that you cannot make the set $M$ of part 2) be non-FC. If such an $M$ and $F$ existed, $f \mid M$ could be extended to a Borel function $g: \mathbb{R} \rightarrow \mathbb{R}$. Then $g$ could be restricted to a co-FC set $N$ so that $g \mid N$ is continuous (this follows from Theorem 2 below). But it would follow that $M \cap N$ is non-FC and $f|(M \cap N)=g|(M \cap N)$ is continuous. This is a contradiction.

EXAMPLE 1.4. Known continuous badly non-differentiable functions, such as the almost nowhere approximately differentiable functions of Jarník [8], show that you cannot make the set $P$ of part 3$)$ be non- $L_{0}$ or non-FC.

Notation. Before discussing the next two examples, we need to define certain notations. $C^{n}$ denotes the $n$ times continuously differentiable functions as usual. $D^{n}$ denotes the $n$ times differentiable functions. " $D^{1}$ " (with the quotes) denotes the functions which are differentiable in the extended sense, i.e. allowing $\pm \infty$ as possible derivative values (continuity of the function is required). " $C$ " denotes those " $D$ " functions $f$ such that the extended real-valued function $f^{\prime}$ is continuous in the extended sense. In general, " $D^{n}$ " denotes those functions $f$ in $C^{n-1}$ such that $f^{(n-1)}$ is in " $D^{1}$ ", and " $C$ " " denotes those functions $f$ in " $D^{n}$ " such that $f^{(n)}$ is continuous in the extended sense. If $0<\alpha \leq 1$, then $\operatorname{Lip}^{\alpha}$ denotes those functions $f$ such that $\left\{[f(x)-f(y)] /\left[|x-y|^{\alpha}\right]\right\}$ is a bounded set, and $C^{1+\alpha}$ denotes those functions $f$ in $C^{1}$ such that $f^{\prime}$ is in $\operatorname{Lip}^{\alpha}$. These properties are related as follows:

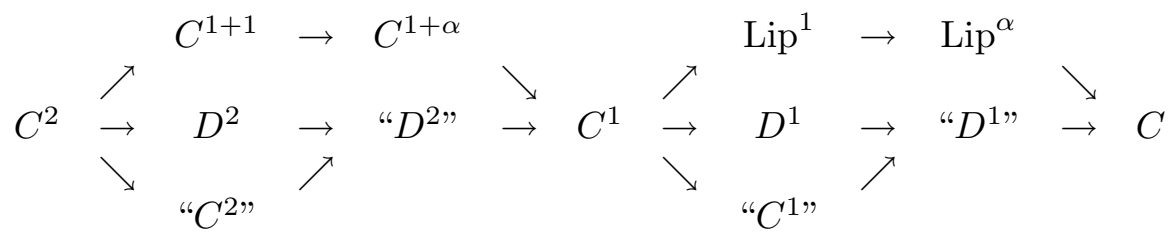

where $C$ denotes the continuous functions. Note that $C^{2}$ and $C^{1+1}$ are not the same. No implications between these properties not indicated in the diagram hold.

EXAMPLE 1.5. The question of whether the function $g$ of 3)(iii) could be made to be $C^{2}$, even for continuous $f$, was stated in [1]. A. Olevskir announced that he had obtained a solution to this problem in his lecture at the 14th Summer Symposium in Real Analysis held in June, 1990. In particular, he announced that there exists a $\operatorname{Lip}^{1} f: \mathbb{R} \rightarrow \mathbb{R}$ such that for every $0<\alpha<1$ and every $g \in C^{1+\alpha}$, the intersection $f \cap g$ is countable. 
[17] is a summary of Olevskil's talk and includes a sketch of how that example is to be constructed. Let $r(x)=1$ for $0<x<1 / 2$ and $r(x)=-1$ for $1 / 2<x<1$, and let $r$ be periodically extended to $\mathbb{R}$. Then, for small enough $q \in(0,1)$ and for rapidly increasing integers $\left\{v_{n}\right\}$, the function

$$
F(x)=\int_{0}^{x} \sum_{n \in \mathbb{N}} q^{n} r\left(v_{n} t\right) d t
$$

will have the desired properties. $\mathbb{N}$ is the set of positive integers. The complete proof of this has not appeared yet, but this author was able to work out a proof to his own satisfaction that the example works, for example, when $q=0.1$ and $v_{n}=n^{n}$ !. We also noticed in the process that $F$ has no uncountable intersection with any $D^{2} g: \mathbb{R} \rightarrow \mathbb{R}$, but that $F$ does have a perfect intersection with a " $C^{2}$ " $g: \mathbb{R} \rightarrow \mathbb{R}$. We now prove this latter fact. We will be primarily interested in the derivative function $f(x)=F^{\prime}(x)$, which is the function defined by the infinite sum inside the integral sign above. This function is defined only at the $x$ 's outside of the countable set of numbers $\left\{k / n^{n} !: k\right.$ an integer, $\left.n \in \mathbb{N}\right\}$. For each $n \in \mathbb{N}$, we will find it convenient to notice that the graph of $f$ lies in the union of a certain collection of $2\left(n^{n} !\right)$ disjoint "boxes" (interiors of rectangles with sides parallel to the axes).

For $n=1$, for example, it is easy to see that the graph of $f$ lies in the union of the two boxes $(0, .5) \times(8 / 90,10 / 90)$ and $(.5,1) \times(-8 / 90,-10 / 90)$. We denote the ordinates of the middles of these two boxes with the generic notation $m_{1}= \pm .1$. The height of both boxes is $h_{1}=2 / 90$ and the width of both boxes is $w_{1}=1 / 2$.

For $n=2,2^{2} ! / 1^{1} !=24$ disjoint boxes are chosen from each of the two boxes of Stage 1, as indicated in Figure 1.

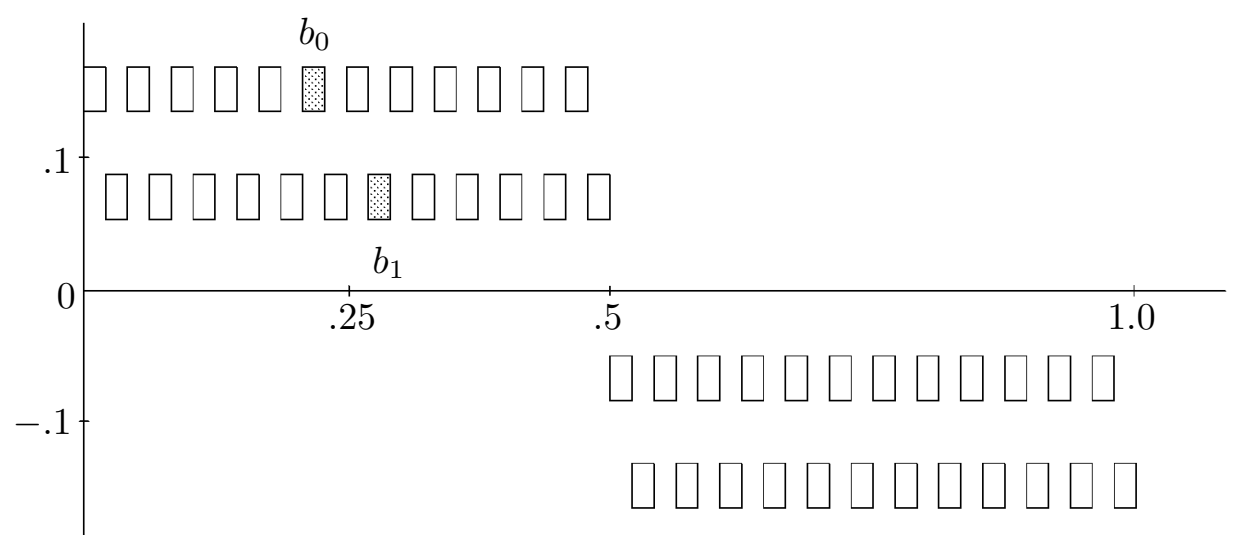

Fig. 1 
We denote the (4 different) ordinates of the middles of the resulting 48 boxes by the generic notation $m_{2}=m_{1} \pm(.1)^{2}$. The height of all 24 boxes is $h_{2}=2(.1)^{2} / 9$ and the width of all 24 boxes is $w_{2}=1 /(2 \cdot 4 !)$.

In general, at Stage $n, n^{n} ! /(n-1)^{n-1}$ ! disjoint boxes are chosen from each of the $2(n-1)^{n-1}$ ! boxes of Stage $n-1$ in a similar fashion to the above. We denote the ( $2^{n}$ different) ordinates of the middles of the resulting $2\left(n^{n} !\right)$ boxes by the generic notation $m_{n}=m_{n-1} \pm(.1)^{n}$. The height of all $2\left(n^{n} !\right)$ boxes is $h_{n}=2(.1)^{n} / 9$ and the width of all boxes is $w_{n}=1 /\left(2 n^{n} !\right)$. Notice that as $n$ increases, the boxes are getting very narrow, in that $\lim _{n \rightarrow \infty} h_{n} / w_{n}=+\infty$.

If for each $n, B_{n}$ denotes the union of the $2\left(n^{n} !\right)$ boxes of Stage $n$, then the graph of $f$ is equal to the set $\bigcap_{n \in \mathbb{N}} B_{n}$.

Now we will describe a perfect set $P \subseteq[0,1]$ and a " $C$ " function $G$ such that $F(x)=G(x)$ for each $x \in P$. We will actually describe the derivative $g=G^{\prime}$ of $G$.

First, we pick two boxes $b_{0}$ and $b_{1}$ (with $X$-projections $I_{0}$ and $I_{1}$, respectively) from among the 24 boxes constructed inside the box $(0, .5) \times$ $(8 / 90,10 / 90)$ in Stage 2 above (see Figure 1). Box $b_{0}$ will be the "upper" box to the left of but closest to the vertical line through the point $x=.25$ and box $b_{1}$ will be the "lower" box to the right of but closest to the same vertical line. In this first step, we can see that $I_{0}=[.25-2 / 48, .25-1 / 48]$ and $I_{1}=[.25+1 / 48, .25+2 / 48]$.

Let $s_{2}$ denote the slope of the slanted line extending from the lower left corner of the box $b_{0}$ to the upper right corner of the box $b_{1}$. Notice that $s_{2}=c\left(2^{2} !\right)(.1)^{2}$, for a negative constant $c$, and that the slope of a line between any point of box $b_{0}$ and any point of box $b_{1}$ is less than $s_{2}$.

Also notice that because of the symmetry and the fractal nature of the graph of $f$, it is true that if $0<\varepsilon<1 / 24, u=(.25-1 / 24)-\varepsilon$, and $v=(.25+1 / 24)+\varepsilon$, then

$$
\int_{u}^{v} f(x) d x=\int_{u}^{v} m_{1} d x, \quad \text { where } m_{1}=.1 .
$$

Next, we will pick boxes $b_{00}$ and $b_{01}$ (with $X$-projections $I_{00}$ and $I_{01}$, respectively) to be two of the $3^{3} ! / 2^{2}$ ! (notice that this is an even integer) boxes constructed at Stage 3 above inside box $b_{0}$. Box $b_{00}$ will be the "upper" box to the left of but closest to the vertical line through the midpoint of $I_{0}$ and box $b_{01}$ will be the "lower" box to the right of but closest to the same vertical line. Boxes $b_{10}$ and $b_{11}$ (with $X$-projections $I_{10}$ and $I_{11}$, respectively) will be chosen in a similar fashion inside box $b_{1}$.

Now, suppose $n>3$ and we have described $I_{d}$ and $b_{d}$ for every binary sequence $d \in\{0,1\}^{n}$. We will pick boxes $b_{d 0}$ and $b_{d 1}$ (with $X$-projections $I_{d 0}$ and $I_{d 1}$, respectively) to be two of the $n^{n} ! /(n-1)^{n-1}$ ! (notice that this 
is an even integer) boxes constructed at Stage $n$ above inside box $b_{d}$. Box $b_{d 0}$ will be the "upper" box to the left of but closest to the vertical line through the midpoint of $I_{d}$ and box $b_{d 1}$ will be the "lower" box to the right of but closest to the same vertical line.

If $d \in\{0,1\}^{n}$, let $s_{n+1}$ denote the slope of the slanted line extending from the lower left corner of the box $b_{d 0}$ to the upper right corner of the box $b_{d 1}$. Notice that $s_{n+1}=c\left((n+1)^{n+1} !\right)(.1)^{n+1}$, for the same negative constant $c$ indicated above, and that the slope of a line between any point of box $b_{d 0}$ and any point of box $b_{d 1}$ is less than $s_{n+1}$.

Also notice that because of the symmetry and the fractal nature of the graph of $f$, it is true that if $0<\varepsilon<1 /\left(2 n^{n} !\right), u$ is distance $\varepsilon$ to the left of the right end of $I_{d 0}$, and $v$ is distance $\varepsilon$ to the right of the left end of $I_{d 1}$, then

$$
\int_{u}^{v} f(x) d x=\int_{u}^{v} m_{n} d x
$$

where $m_{n}$ is the ordinate of the midpoint of box $b_{d}$.

The perfect set $P=\left(I_{0} \cup I_{1}\right) \cap\left(I_{00} \cup I_{01} \cup I_{10} \cup I_{11}\right) \cap \ldots$, and the function $g(x)$ is defined to be equal to the function $f(x)$ for $x \in P$. Suppose $(u, v)$ is a segment contiguous to the perfect set $P$. There is a first $n$ such that $u$ and $v$ are separated by intervals $I_{d 0}$ and $I_{d 1}$ at Stage $n$ (i.e. for some $\left.d \in\{0,1\}^{n}\right)$. Then the point $(u, g(u))$ is on the lower edge of box $b_{d 0}$ and the point $(v, g(v))$ is on the upper edge of box $b_{d 1}$. Furthermore, $u$ is the same distance to the left of the right end of $I_{d 0}$ as $v$ is to the right of the left end of $I_{d 1}$. We define $g$ on the interval $[u, v]$ as follows. Connect the point $(u, g(u))$ to the point $(v, g(v))$ with a decreasing " $C$ " " function $g$ which has derivative $-\infty$ at both $u$ and $v$, has derivative less than $s_{n} / 2$ at every point of $[u, v]$, and which is symmetric about the point midway between $(u, g(u))$ and $(v, g(v))$. This can be accomplished by piecing together two small circular arcs emanating from the two end points, and then connecting the arcs by a straight line. It follows from the symmetry that

$$
\int_{u}^{v} g(x) d x=\int_{u}^{v} f(x) d x=\int_{u}^{v} m_{n} d x,
$$

where $m_{n}$ is the ordinate of the middle point of box $b_{d}$.

The function $g$ is defined to the left of the left end of the perfect set $P$ and to the right of the right end of $P$ to be " $C$ " " and to make $F$ and $G$ match up at the left end of $P$. It follows that $F(x)=G(x)$ for every $x \in P$.

It also follows from the construction of $g$ that $g^{\prime}(x)=-\infty$ for every $x \in P$, that $g$ is differentiable in the ordinary sense for all other $x$, and that $g$ is " $C$ ", so that $G$ is " $C$ ". This completes the argument. 
The above construction has led the author to believe that the following is probably true.

Conjecture 1.6. For every continuous $f: \mathbb{R} \rightarrow \mathbb{R}$, there exists a " $C$ " $g: \mathbb{R} \rightarrow \mathbb{R}$ such that $f \cap g$ is uncountable.

We are not at this stage able to provide a proof of this conjecture. By contrast we are able to give the following example, which shows that the function $g$ of 3 )(iii) cannot be made to be " $C$ " (or even " $D^{2}$ ").

EXAMPLE 1.7. There exists a $B^{1}$ function $f: \mathbb{R} \rightarrow \mathbb{R}$ which has no uncountable intersection with any " $D^{2}$ " $g: \mathbb{R} \rightarrow \mathbb{R}$. We will piece together countably many copies of the example constructed in the proof of Theorem 22 of [1], which states that for every $\varepsilon>0$ there exists a perfect set $P \subseteq[0,1]$ with $\lambda(P)>1-\varepsilon$ and a continuous $f: P \rightarrow \mathbb{R}$ such that $f \cap g$ is finite for every $D^{2} g:[0,1] \rightarrow \mathbb{R}$. It was pointed out in [3] that this function $f$ also has the property that $\{x: f(x)=g(x)\}$ has no bilateral limit points and at most finitely many one-sided limit points for every " $D$ " $g:[0,1] \rightarrow \mathbb{R}$.

Now, to begin the construction. For each $[a, b] \subseteq[0,1]$, let $P[a, b]$ be a perfect subset $P$ of $[a, b]$ with $\lambda(P)=(b-a) / 2$, and let $f[a, b]: P \rightarrow \mathbb{R}$ be a continuous function $f: P \rightarrow \mathbb{R}$ with the properties described above. Also assume that $f[a, b]$ is increasing and non-negative (as is the case in the example of [1]). Let $P_{1}=P[0,1]$ and $f_{1}=f[0,1]$. List the components of $[0,1] \backslash P_{1}$ as $\left\{\left(a_{1, j}, b_{1, j}\right): j=1,2, \ldots\right\}$. For each $j$, let $P_{1, j}=P\left[a_{1, j}, b_{1, j}\right]$, let $f_{1, j}=f\left[a_{1, j}, b_{1, j}\right]$, and let $Q_{2}=P_{1} \cup P_{1,1} \cup P_{1,2} \cup \ldots$ For the $i$ th step, assume $P_{i-1, j}$ and $f_{i-1, j}$ have been defined for all $j$, and $Q_{i}=Q_{i-1} \cup$ $P_{i-1,1} \cup P_{i-1,2} \cup \ldots$ List the components of $[0,1] \backslash Q_{i}$ as $\left\{\left(a_{i, j}, b_{i, j}\right): j=\right.$ $1,2, \ldots\}$. For each $j$, let $P_{i, j}=P\left[a_{i, j}, b_{i, j}\right]$, let $f_{i, j}=f\left[a_{i, j}, b_{i, j}\right]$, and let $Q_{i+1}=Q_{i} \cup P_{i, 1} \cup P_{i, 2} \cup \ldots$ Let $G=[0,1] \backslash\left(Q_{2} \cup Q_{3} \cup \ldots\right)$. $G$ is a dense $G_{\delta}$ of measure zero. Let $g:[0,1] \rightarrow \mathbb{R}$ be a continuous increasing nonnegative function such that $g^{\prime}(x)=+\infty$ for every $x \in G$. Reorder and rename $\left\{\left(P_{i, j}, f_{i, j}\right): i, j=1,2, \ldots\right\}$ as $\left\{\left(P_{i}, f_{i}\right): i=2,3, \ldots\right\}$. For each $i$, let $g_{i}:[0,1] \rightarrow \mathbb{R}$ be a $C^{\infty}$ function such that

$$
g(x)+1 /(i+1)<g_{i}(x)<g(x)+1 / i
$$

for every $x \in[0,1]$ and let $\delta_{i}>0$ be small enough that

$$
g(x)+1 /(i+1)<g_{i}(x)+\delta_{i} f_{i}(x)<g(x)+1 / i
$$

for every $x \in P_{i}$. Let $f(x)=g(x)$ if $x \in G$, and let $f(x)=g_{i}(x)+\delta_{i} f_{i}(x)$ if $x \in P_{i}$. The function $f$ is $B^{1}$ (in fact upper semicontinuous). Suppose there exists a " $D$ " function $h:[0,1] \rightarrow \mathbb{R}$ such that the set $M=\{x:$ $f(x)=g(x)\}$ is uncountable. Let $Q$ be a perfect subset of $M$. Since $f$ is differentiable on $M$ and $g^{\prime}=+\infty$ on $G, Q \cap G$ is finite. Then there is an $i$ 
such that $Q \cap P_{i}$ is uncountable. Thus, it follows that $f_{i}$ has an uncountable intersection with the " $D$ " function $\left(h-g_{i}\right) / \delta_{i}$, which is a contradiction.

3. Functions with the Baire property. The second most familiar theorem of the type we are considering here is part 1) of the following for "functions with the Baire property", which we are calling the $B_{\mathrm{w}}$-measurable functions.

Theorem 2. If $f: \mathbb{R} \rightarrow \mathbb{R}$ is $B_{\mathrm{w}}$-measurable, then

1) there exists a co-FC set $M$ such that $f \mid M$ is continuous ([16] and [9]),

2) there exists a co-FC set $M$ and a $D^{1}$ function $F$ such that $f \mid M=$ $F^{\prime} \mid M$,

3) there exists a perfect set $P$ such that $f \mid P$

(i) is monotonic [7],

(ii) is " $D^{1}$ " (relative to $P$ ) [5],

(iii) $=g \mid P$ for some " $C^{1 "} " g: \mathbb{R} \rightarrow \mathbb{R}[3]$.

Proof. Part 1) is sometimes called the Nikodym-Kuratowski Theorem and the proof can be found in [16] and [9]. Part 2) is new and the proof will follow easily from Theorem 4.14 of the paper [18] of Petruska and Laczkovich. That theorem implies that if $\lambda(M)=0$ and $f=g \mid M$ for some $B^{1} g$ : $[0,1] \rightarrow \mathbb{R}$, then $f=h \mid M$ for some derivative $h:[0,1] \rightarrow \mathbb{R}$. To prove 2 ), first let $M_{1}$ be the set from part 1) of the theorem. Then, let $M$ be a co-FC $G_{\delta}$ subset of $M_{1}$ such that $\lambda(M)=0 . \quad f \mid M$ can be extended to a $B^{1} g:[0,1] \rightarrow \mathbb{R}$. It follows from the result quoted above that $f \mid M=$ $g|M=h| M$ for some derivative $h:[0,1] \rightarrow \mathbb{R}$. Then let $F$ be a $D^{1}$ function such that $F^{\prime}=h$. This proves part 2). Part 3) was not stated explicitly for $B_{\mathrm{w}}$-measurable functions originally, but follows from part 1) and the results concerning continuous functions in the papers referenced above. Note that (iii) of part 3) actually implies (ii) of part 3), which was not the case in Theorem 1.

EXAMPLE 2.1. It is known that you cannot make the set $M$ of part 1) non- $L_{0}$, even (assuming $\mathrm{CH}$ ) for a $B_{\mathrm{r}}$-measurable $f$ such as that described in Example 2 of [4]. That same example shows that you cannot make the set $M$ of part 2) be non- $L_{0}$ and have a continuous $F:[0,1] \rightarrow \mathbb{R}$ such that $F^{\prime}(x)=f(x)$ for every $x \in M$. If such an $M$ and $F$ existed, $f \mid M$ could be extended to a Borel function $g: \mathbb{R} \rightarrow \mathbb{R}$. Then $g$ could be restricted to a set $N$ of large measure so that $g \mid N$ is continuous. But if you make $\lambda(N)$ large enough, it would follow that $M \cap N$ is non- $L_{0}$ and $f|(M \cap N)=g|(M \cap N)$ is continuous. This is a contradiction.

EXAMPLE 2.2. We cannot remove the quotation marks from (ii) and (iii) of part 3) because it was shown in Example 3.1 of [3] that there is a 
$B_{\mathrm{w}}$-measurable $f:[0,1] \rightarrow \mathbb{R}$ with no $D^{1}$ restriction to any perfect set. We now show that $\mathrm{CH}$ implies the existence of a $B_{\mathrm{r}}$-measurable function which has this property. Assume $\mathrm{CH}$, and let $\left\{P_{\alpha}: \alpha<\omega_{1}\right\}$ be a well-ordering of the perfect subsets of $[0,1]$. Let $H_{1}$ be a $G_{\delta} L_{0}$ relatively dense subset of $P_{1}$. Let $G_{1}=H_{1}$. Let $f_{1}$ be a continuous increasing function with domain $G_{1}$ such that $f^{\prime}(x)=+\infty$ for every $x \in G_{1}$. Now suppose that $\alpha<\omega_{1}$, and $H_{\beta}, G_{\beta}$, and $f_{\beta}$ have been defined for every $\beta<\alpha$ (where the $H_{\beta}$ are $G_{\delta}$ sets). For each $\beta<\alpha$, let $O_{\alpha, \beta}$ be the maximal relatively open subset of $P_{\alpha}$ in which $H_{\beta}$ is dense $\left(O_{\alpha, \beta}\right.$ might be empty) and let $U_{\alpha}$ denote the relative interior of $P_{\alpha} \backslash \bigcup_{\beta<\alpha} O_{\alpha, \beta}$ (U $U_{\alpha}$ may be empty). Note that the $\left\{O_{\alpha, \beta}: \beta<\alpha\right\}$ must be disjoint and that $\bigcup_{\beta<\alpha} H_{\beta}$ is relatively first category in $U_{\alpha}$. Thus, we can let $H_{\alpha}$ be a $G_{\delta} L_{0}$ relatively dense subset of $U_{\alpha} \backslash \bigcup_{\beta<\alpha} H_{\beta}$. We can let $G_{\alpha}=H_{\alpha} \cup\left[\bigcup_{\beta<\alpha}\left(H_{\beta} \cap O_{\alpha, \beta}\right)\right]$, and let $f_{\alpha}$ be a function defined on $G_{\alpha}$ which is continuous increasing with $f^{\prime}(x)=+\infty$ on $H_{\alpha}$ and which agrees with $f_{\beta}$ on $H_{\beta} \cap O_{\alpha, \beta}$ for $\beta<\alpha$. Let $f$ be the union of the $f_{\alpha}$ on the union of the $H_{\alpha}$, and let $f$ be arbitrarily defined on the rest of $[0,1]$. It is clear that if $P=P_{\alpha}$ is any perfect subset of $[0,1]$, then $G_{\alpha}$ is a dense relatively $G_{\delta}$ subset of $P$ such that $f \mid G_{\alpha}$ is continuous. This is equivalent to $f$ being $B_{\mathrm{r}}$-measurable (see [16]). It is also clear that no $f \mid P_{\alpha}$ is $D^{1}$ because $f \mid G_{\alpha}$ will have derivative identically equal to $+\infty$.

4. Marczewski measurable functions. The theorem for $(s)$-measurable (or "Marczewski measurable") functions is the following.

Theorem 3. If $f: \mathbb{R} \rightarrow \mathbb{R}$ is (s)-measurable, then

1 ) there exists a perfectly dense subset $M$ of $\mathbb{R}$ (every open subset of $\mathbb{R}$ contains a perfect subset of $M)$ such that $f \mid M$ is continuous [4],

2) there exists a perfectly dense subset $M$ of $\mathbb{R}$ and a $D^{1}$ function $F$ : $\mathbb{R} \rightarrow \mathbb{R}$ such that $f\left|M=F^{\prime}\right| M$,

3) $\{$ same as 3) of Theorem 2$\}$.

Pro of. Part 1) was proved in [4]. To prove 2) we could take the set $M_{1}$ of part 1) and let $M$ be a perfectly dense subset of $M_{1}$ such that $\lambda(M)=0$. Then $f \mid M$ could be continuously extended to a $G_{\delta}$ set, and that function could then be extended to a $B^{1} g:[0,1] \rightarrow \mathbb{R}$. Then we can call on the result of Petruska and Laczkovich used earlier to conclude that $f|M=g| M=h \mid M$ for some derivative $h:[0,1] \rightarrow \mathbb{R}$. Then, let $F$ be a primitive of $h$, and the proof of part 2) is obtained. Again, part 3) was not originally stated for $(s)$-measurable functions but the proof follows from part 1) and the results concerning continuous functions which appear in the references given.

EXAMPLE 3.1. It is known that you cannot make the set $M$ of part 1) be non- $L_{0}$ or non-FC (assuming $\mathrm{CH}$ ) for $(s)$-measurable $f$ such as that 
described in Example 3 of [4]. That same example shows that you cannot make the set $M$ of part 2) be non- $L_{0}$ or non-FC and have a continuous $F:[0,1] \rightarrow \mathbb{R}$ such that $F^{\prime}(x)=f(x)$ for every $x \in M$. The reasoning is similar to that of Example 1.3 and Example 2.1.

EXAMPLE 3.2. It was already pointed out in Example 1.1 that you cannot make the set $M$ of part 1$)$ be co- $\left(s^{0}\right)$, even if $f$ is a $B^{1}$ function. Neither can you make the set $M$ be co- $\left(s^{0}\right)$ and have a continuous $F:[0,1] \rightarrow \mathbb{R}$ such that $F^{\prime}(x)=f(x)$ for every $x \in M$, even for the $B^{1}$ function $f(x)=1 / x$ (with $f(0)=0)$. If such an $F$ and $M$ existed, then $(F-\operatorname{Ln})^{\prime}(x)=0$ on a co- $\left(s^{0}\right)$ set (Ln is the natural log function on $\left.(0,1]\right)$. But since the set where $(F-\mathrm{Ln})^{\prime}$ exists is a Borel set, it follows that $(F-\mathrm{Ln})^{\prime}$ exists and is zero on a co-countable set. It therefore follows that $F-\operatorname{Ln}$ is constant on $(0,1]$, but this contradicts the assumption that $F$ is continuous on $[0,1]$.

\section{References}

[1] S. Agronsky, A. M. Bruckner, M. Laczkovich and D. Preiss, Convexity conditions and intersections with smooth functions, Trans. Amer. Math. Soc. 289 (1985), 659-677.

[2] H. Blumberg, New properties of all real functions, ibid. 24 (1922), 113-128.

[3] J. B. Brown, Differentiable restrictions of real functions, Proc. Amer. Math. Soc. 108 (1990), 391-398.

[4] J. B. Brown and K. Prikry, Variations on Lusin's theorem, Trans. Amer. Math. Soc. 302 (1987), 77-86.

[5] A. M. Bruckner, J. G. Ceder and M. L. Weiss, On the differentiability structure of real functions, ibid. 142 (1969), 1-13.

[6] J. Ceder, Some examples on continuous restrictions, Real Anal. Exchange 7 (1981/ 82), $155-162$.

[7] F. Filipczak, Sur les fonctions continues relativement monotones, Fund. Math. 58 (1966), 75-87.

[8] V. Jarník, Sur les nombres dérivés approximatifs, ibid. 22 (1934), 4-16.

[9] C. Kuratowski, La propriété de Baire dans les espaces métriques, ibid. 16 (1930), 390-394.

[10] K. Kuratowski and A. Mostowski, Set Theory with an Introduction to Descriptive Set Theory, North-Holland, Amsterdam 1976.

[11] M. Laczkovich, Differentiable restrictions of continuous functions, Acta Math. Hungar. 44 (1984), 355-360.

[12] N. Lusin, Sur les propriétés des fonctions mesurables, C. R. Acad. Sci. Paris 154 (1912), 1688-1690.

[13] —, Sur la recherche des fonctions primitives, ibid. 162 (1916), 975-978.

[14] E. Marczewski (Szpilrajn), Sur une classe de fonctions de M. Sierpiński et la classe correspondante d'ensembles, Fund. Math. 24 (1935), 17-34.

[15] - Sur les ensembles et les fonctions absolument mesurables, C. R. Soc. Varsovie 30 (1937), 39-68.

[16] O. Nikodym, Sur la condition de Baire, Bull. Internat. Acad. Polon. 1929, 591598. 
[17] A. Olevskiŭ, Some interpolation problems in real and harmonic analysis, Real Anal. Exchange 16 (1990/91), 353-361.

[18] G. Petruska and M. Laczkovich, Baire 1 functions, approximately continuous functions and derivatives, Acta Math. Hungar. 25 (1974), 189-212.

[19] W. Sierpiński, Sur un problème de M. Ruziewicz concernant les superpositions de fonctions jouissant de la propriété de Baire, Fund. Math. 24 (1935), 12-16.

DIVISION OF MATHEMATICS

AUBURN UNIVERSITY

AUBURN, ALABAMA 36849-5310

U.S.A.

Received 28 June 1991;

in revised form 5 November 1991 